were hepatitis $\mathrm{B} / \mathrm{C} \quad(72.2 \%)$ and alcohol consumption $(10.0 \%)$. Fifty-two $(20.1 \%)$ patients were on the treatment of NSBBs and $53(20.5 \%)$ patients were treated with NSBBs before, whereas 23 patients $(8.9 \%)$ were with contraindications. Thirteen patients (25.0\%) achieved hemodynamic response and the target dose was $32.1 \mathrm{mg} / \mathrm{d}$ for propranolol and $12.5 \mathrm{mg} / \mathrm{d}$ for carvedilol respectively. Overall adverse effects (AEs) were substantially more prevalent in endoscopic therapy than in NSBBs therapy $(61.4 \%$ vs $25.7 \%, P<0.001)$, but severe AEs leading to therapy cessation were more prevalent in NSBBs therapy $(12.4 \%$ vs $5.2 \%, P<0.001)$. During the 6-month follow-up, $53.8 \%$ of patients on NSBBs showed good compliance and $59.0 \%$ of patients on endoscopic therapy showed good compliance $(P=0.490)$. Upon multivariate analysis, only old age and high work intensity were associated with poor drug compliance, while education background, healthcare insurance, AEs, drug dose and disease severity did not affect the compliance.

Conclusions Clinical use of NSBBs for cirrhotic patients is far from optimal considering the low prevalence and high proportion of ineffective low-dose. NSBBs medication bears a higher rate of severe AEs compared with endoscopic therapy. Therapy compliance of both NSBBs and endoscopy are unsatisfactory, and optimized follow-up management is greatly needed.

\section{IDDF2020-ABS-0050 SPIRAL CT IN THE CLINICAL SIGNIFICANCE OF PORTAL CAVERNOUS CHANGE IN HEPATOCELLULAR CARCINOMA}

Yi Y Y*. Department of Critical Care Medicine, The Second Affiliated Hospital of Guangzhou University of Chinese Medicine, China

\subsection{6/gutjnl-2020-IDDF.136}

Background Cavernous degeneration of portal vein (cavernous transformation of portal vein, CTPV) refers to the formation of a large number of collateral branches or recanalization after complete or partial obstruction of the main portal vein or its branches. To investigate the value of spiral CT dual phase scanning for diagnosis of portal cavernous degeneration of liver cancer.

Methods 103 cases of hepatocellular carcinoma with portal cavernous change were enrolled from the First Affiliated Hospital of Sun Yat-sen University between January 01, 2015, to December 31, 2018. The abdominal CT data were analyzed, including blood vessel cross-sectional features, hepatic parenchyma perfusion, CT findings and features of portal hypertension.

Results The main manifestations of CT were several small blood vessels around the portal vein in the occlusion position, the heavy ones were beaded, and the hepatic parenchyma was transient perfusion abnormality in the arterial phase, showing a zonal high-density shadow around the liver, the main findings were as follows: At the arterial stage, the hepatic parenchyma was abnormal. In the portal phase, the whole liver showed uniform density. In cirrhotic patients with portal hypertension, collateral circulation vessels (27/103), ascites (18/ $103)$, splenomegaly (45/103) were seen around the hilum of the spleen.

Conclusions Spiral CT dual-phase scanning is an effective method for diagnosis of portal cavernous degeneration of liver cancer, which can provide a necessary basis for clinical treatment and prognosis.

\section{IDDF2020-ABS-0051 ASSOCIATION BETWEEN TYPE-2 DIABETES MELLITUS AND PLATELET DISTRIBUTION WIDTH IN PATIENTS WITH PRIMARY LIVER CANCER}

Yi Yu*. Department of Critical Care Medicine, The Second Affiliated Hospital of Guangzhou University of Chinese Medicine, China

10.1136/gutjnl-2020-IDDF.137

Background To investigate the association between type-2 diabetes mellitus (T2DM) and platelet distribution width (PDW) in patients with primary liver cancers (PLC).

Methods We included 387 patients with a hospital discharge diagnosis of PLC from January 2010 to January 2017 in the MIMIC-III database were analyzed retrospectively. Demographic and clinical characteristics data were analyzed. The multivariate logistic regression model was used to determine the association between T2DM and PDW in PLC patients.

Results Of the total 387 patients, 105 (27.1\%) cases were diagnosed with T2DM, the mean hemoglobin was (113.7 $\pm 12.8) \mathrm{g} / \mathrm{L}$, the median/interquartile range of PDW was $15.2 \%$ / (11.1\%-17.3\%). A statistical difference was found by univariate analysis for the PDW $[11.0 \% /(8.6 \%-12.4 \%)$ vs. $16.7 \% /$ (15.4-18.0\%), $\mathrm{P}=0.037]$. The two groups, with a statistical difference $(\mathrm{OR}=0.437,95 \% \mathrm{CI}: 0.236-0.638, \mathrm{P}=0.04)$ was found by the multivariate analysis, after controlling the age, HBV infection, hemoglobin.

Conclusions PLC patients with T2DM have obviously decreased PDW, compared with those without T2DM, which is modified by other factors.

\section{IDDF2020-ABS-0052 CLINICAL SIGNIFICANCE OF THE SERUM LEPTIN LEVELS OF HEPATITIS C PATIENTS AND THE BLOOD LIPID LEVELS DETECTION}

Yi Yu*. Department of Critical Care Medicine, The Second Affiliated Hospital of Guangzhou University of Chinese Medicine, China

\subsection{6/gutjnl-2020-IDDF.138}

Background To investigate the correlation between the serum leptin levels and blood lipids levels detection in patients with hepatitis $\mathrm{C}$.

Methods 118 patients with hepatitis C in our hospital were randomly selected as the experimental group, while 128 cases of the healthy check-up as the control group. The serum leptin levels and blood lipids levels of all the subjects were detected, and their results were statistically analyzed.

Results The average results of all the levels in the experimental groups were higher than that those of the control group. Serum leptin was increased significantly in patients with hepatitis C compared with the healthy controls $(23.17 \pm 6.46) \mathrm{ng} /$ $\mathrm{ml}$ vs $(5.47 \pm 2.71) \mathrm{ng} / \mathrm{ml}, \mathrm{P}=0.01)$. TC in the hepatitis $\mathrm{C}$ group increased significantly compared with that in the control group, which was of significant difference $(\mathrm{P}=0.01)$; HDL-C decreased more obviously in the hepatitis $\mathrm{C}$ group, which was of statistical difference $(\mathrm{P}=0.01)$.

Conclusions Through the comprehensive indexes of the serum leptin levels and the combined detection of blood lipid in patients with Hepatitis C liver disease, it can accurately reflect the severity of the hepatitis $\mathrm{C}$ liver disease, and it is of guidance significance in clinical diagnosis and treatment. 